\title{
Simplified protocol for horizontal and vertical post-extractive GBR with intentionally exposed PTFE membrane- Case series
}

\section{Cappellin MR}

\section{Affiliations:}

Clinica dentale Cappellin, Benefit Corporation (Pinerolo, Turin - ITALY

\section{Corresponding author:}

Mario R. Cappellin

mario.cappellin@clinicacappellin.it

\section{ABSTRACT}

Purpose: Post-extractive sites often need soft and hard tissue regeneration in order to place implants with optimal functional and aesthetic conditions. The author proposed several techniques for bone preservation and regeneration: most of them requires release incisions and coronally advanced flap to obtain primary closure, so regain a correct alignment of keratinized gingiva makes mandatory a further surgery with a connective tissue graft from secondary surgical site.

Case report: Since May 2018 we applied with some adjustments Open Barrier Technique (proposed by E. Funakoshi, 2005) in 152 post-extractive alveolar preservation and GBR; after 3-6 months we placed 194 implants, with 100\% success and survival rate.

Conclusions: Our simplified protocol allows performing vertical and horizontal GBR in post-extractive sites without release incisions: non resorbable PTFE membrane protects wound and bone graft for 6-8 week; secondary healing ensures thick keratinized tissue and bone maturation, suitable to place implants after 3-6 months.

\section{KEYWORDS}

Alveolar preservation, horizontal and vertical GBR, PTFE membrane intentionally exposed, modified Open Barrier Technique 


\section{INTRODUCTION}

Our principal aim is to validate a simplified protocol for bone regeneration in post-extractive sites, avoiding vertical release incisions and flap displacement, in order to obtain new keratinized gingiva (Figure 4,11) by secondary closure of the surgical site and correct bone volume to place prosthetically planned implants.

We used Open Barrier Technique (E. Funakoshi) with intentionally exposed d-PTFE (Cytoplast) membrane to protect the bone graft (Geistlich Bio-Oss ${ }^{\circ}$ with A-PRF and PRGF) 1,2,3,4,5,8,9; membranes have nanopores less than 0,3 microns to prevent bacterial infiltration and epithelium colonization of bone graft, but allow microscopical perfusion of oxygen and nutrition. ${ }^{6,7}$ We used cylinder-conical implants (Legacy ${ }^{\mathrm{TM}}$ by Implant Direct ${ }^{\circ}$ and JD Evolution Plus ${ }^{\circ}$ by JDentalCare ${ }^{\circ}$ ) with lengths from $10 \mathrm{~mm}$ to $15 \mathrm{~mm}$ and diameter from $3.7 \mathrm{~mm}$ to $5.7 \mathrm{~mm}$; abutments for provisional and definitive prostheses were made with CAD-CAM customized transmucosal profile (anodized titanium).

As sutures, we use PTFE 4/0, which can be maintained in place for 2-3 weeks without inflammation and plaque retention.

Postoperative therapy provides 5 days of antibiotic and analgesic therapy; we add Herboplanet Bacsol (2 tablets twice a day for 30 days and 1 tablet twice a day for 30 more days, with Echinacea angustifolia, Salix alba, Harpagophytum procumbens, Origanum vulgare, Thymus vulgaris, Satureja montana, Cinnamomum zeylanicum, to help and stimulate the immune system).

\section{Protocol}

After tooth extraction, bone surface must be accurately cleaned to remove granulation tissue; the site should have at least one interproximal residual bone wall, but for simpler membrane stabilization two walls are recommended.

Vestibular and lingual/palatal soft tissue must be detached (without any vertical incision) as periosteal elevator surpasses the residual bone for $3-4 \mathrm{~mm}$ in depth: the membrane will be shaped in order to be inserted in these spaces and to cover the whole graft plus 2-4mm in every direction. Horizontal periosteal incisions are not provided, the soft tissues can be mobilized with the Soft Brushing Technique (J. Choukroun), disorganizing the periosteal collagen fiber as the brush tools don't cut anything avoiding conventional flap: the aim is to decrease the tension and increase the release of the tissues, which have as more as possible to cover the new graft volume.

PTFE membrane should be inserted first in lingual/palatal site, since usually is simpler; a bone graft must be fit in place, obtaining it by heterologous bone mixed with A-PRF (cut into small pieces) and solidifying with PRGF ${ }^{10,11,12,13}$, very handy and stable to be positioned in desired volume, overcompensating by $2-3 \mathrm{~mm}$.

Then the membrane is curved over the graft and placed to cover whole vestibular dimension; it is very important that the membrane does not touch adjacent teeth, leaving almost $1-2 \mathrm{~mm}$ from dental surface.

Our protocol provides A-PRF fibrin membranes (Figure 12) over PTFE membrane and especially it's recommended if the site left for secondary healing has a diameter bigger than 6-8 mm, to ensure additional protection against bacteria in the first days of healing.

The sutures must be placed from vestibular to lingual/palatal, always in the mesial and distal portion and then one stitch every $2-3 \mathrm{~mm}$, regarding total extension of extraction site; a final horizontal mattress suture under the vestibular mucogingival line can be used to obtain a stress-breaker effect.

For the first 6-8 weeks, we prescribed Chlorhexidine Gluconate $0,20 \%$ after meals and before sleep; every 8-10 days we recommend to gently clean exposed portion of the membrane with hydrogen peroxide on cotton, to remove plaque (Figure 1 , $6,8)$, but it is not mandatory: 14 patients didn't strictly comply, follow up appointments and we registered no significant differences, so now we use to recall patients once after 7 days and then every 2-3 week in the first 2 months (Figure 13).

Regarding the area of exposed membrane, after 6-8 weeks its aspect changes from opaque white to transparent grey (Figure 8), so it can be removed with tweezers: the membrane is very simple to pull and usually this step doesn't require anesthesia. Under the membrane the granulation tissue (Figure 2, 7, 9, 14) is immature, but it strongly protects the graft and mainly in 3-4 months is totally epithelialized (Figure $3,10,15)$, with a very remarkable tissue thickness (Figure 4).
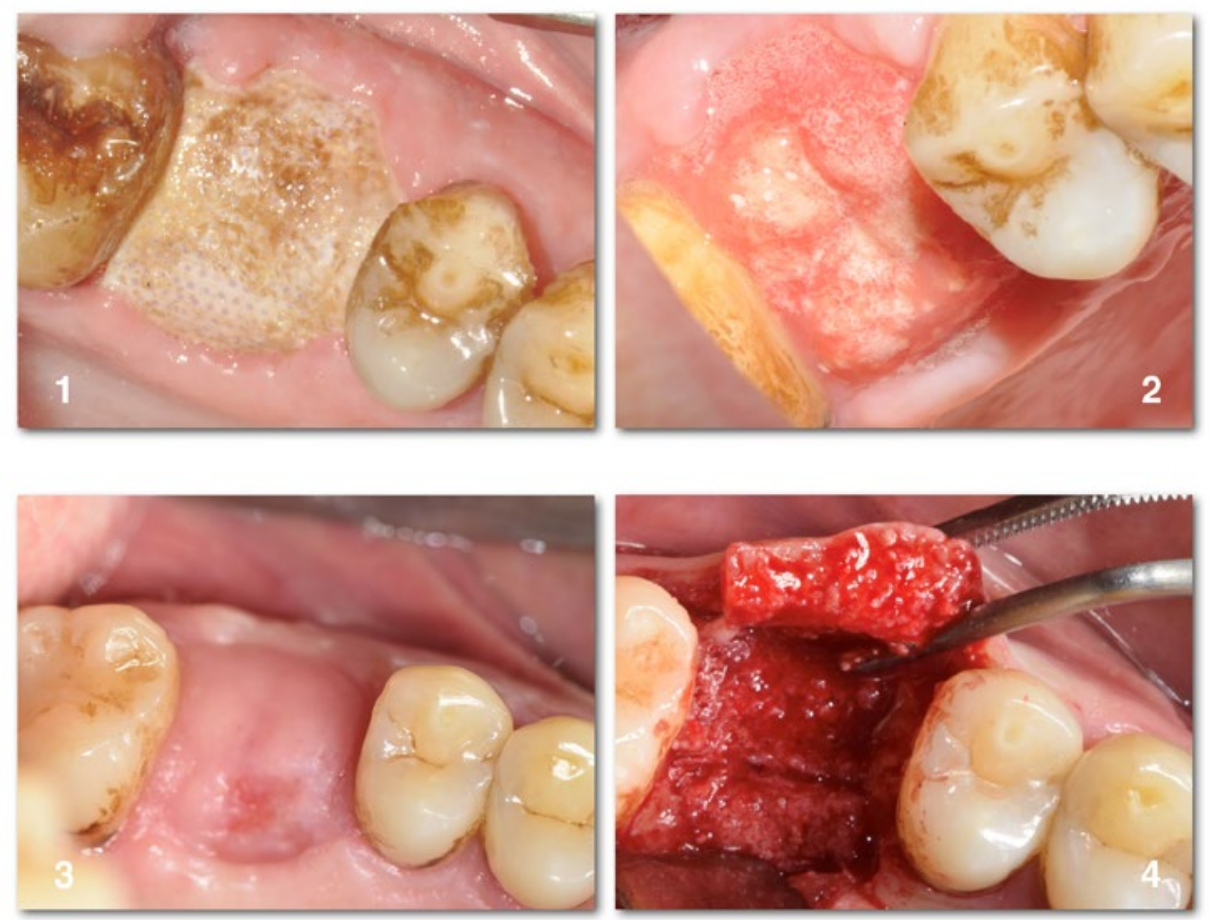

Figures1-4. Healing evolution: 2 weeks / 6 weeks / 6 months (before and after second surgery) 
Figures 5-6-7 show wound evolution: sutures close partially tissues; then secondary healing gradually enlarges exposed portion of the PTFE membrane; notice the healthy aspect of keratinized tissues that surround the membrane. It is important that no PTFE border becomes exposed: in this case membrane must be immediately removed, even in the early phase (we are exposing complications in "results and conclusion" section).
Regarding graft volume, after 3-6 months a CBCT is recommended to plan implant(s) prosthetically guided in the second surgery: thus far, bone isn't mature, but its consistency is enough to reach optimal primary stability (in present study minimum torque insertion was $23 \mathrm{~N}$, maximum $63 \mathrm{~N}$, average $46 \mathrm{~N}$ ) and it appears bloody (Figure 16).

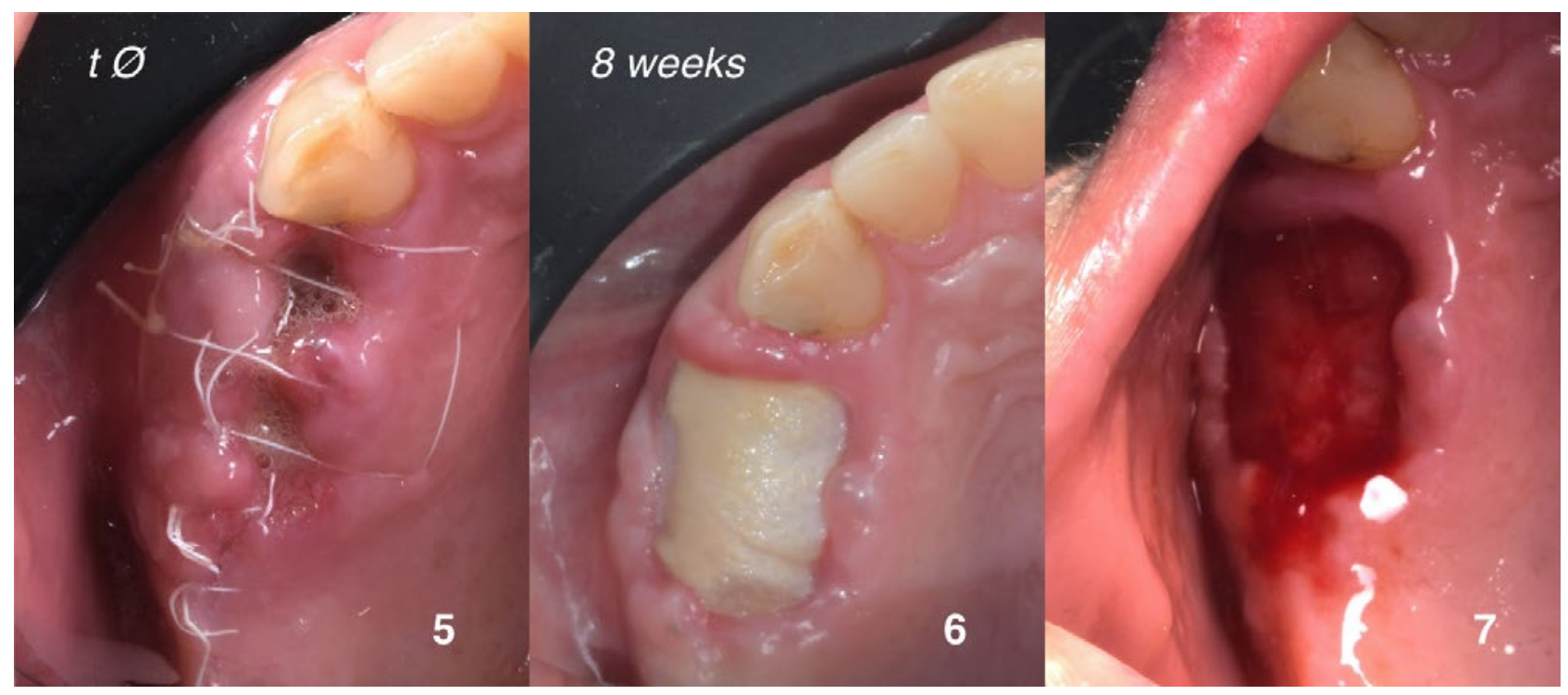

Figures 5-7. Healing evolution: after the first surgery / 8 weeks (before and after removing the membrane)
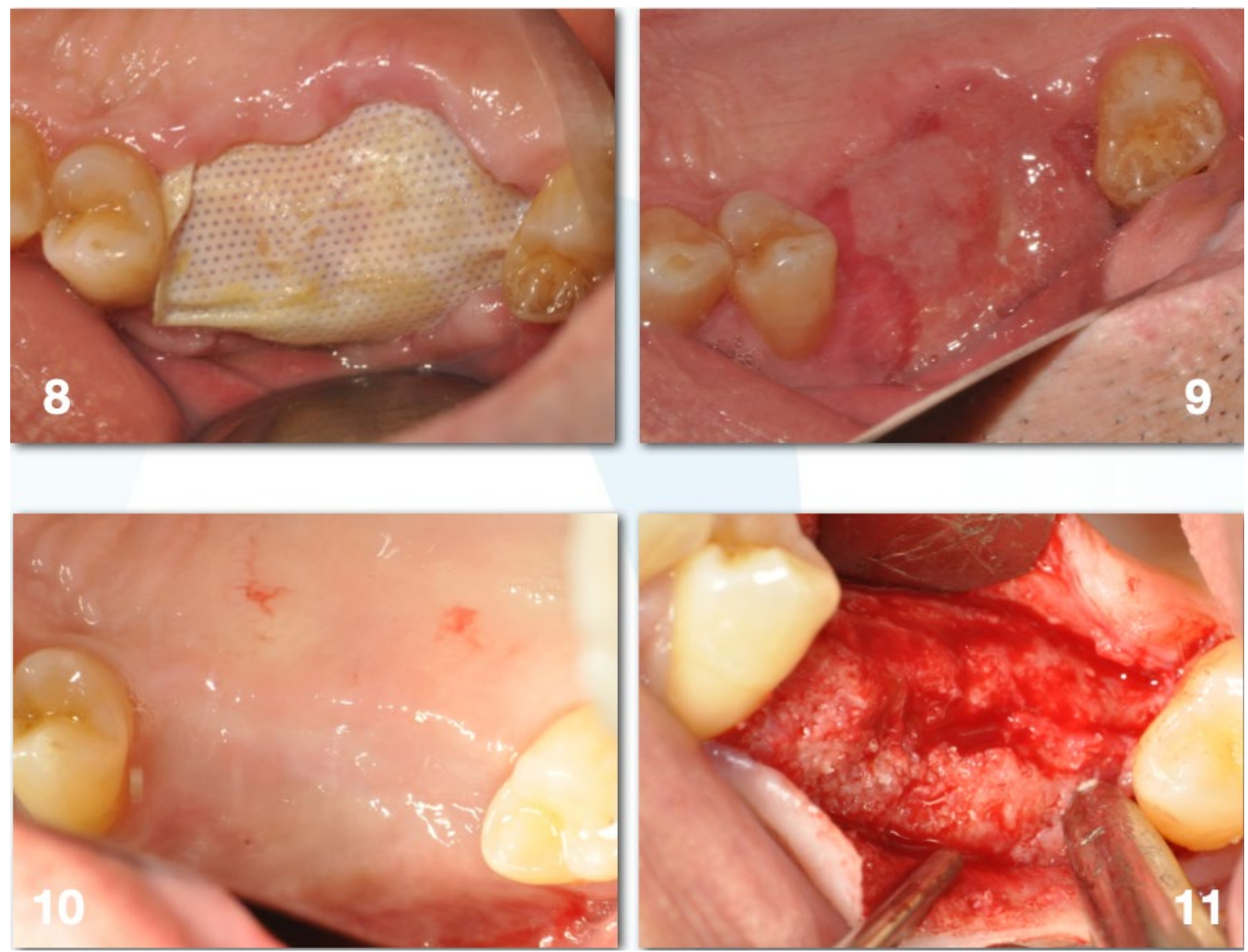

Figures 8-11. Healing evolution: 7 weeks (before and after removing the membrane) / 6 months (before and after second surgery) 

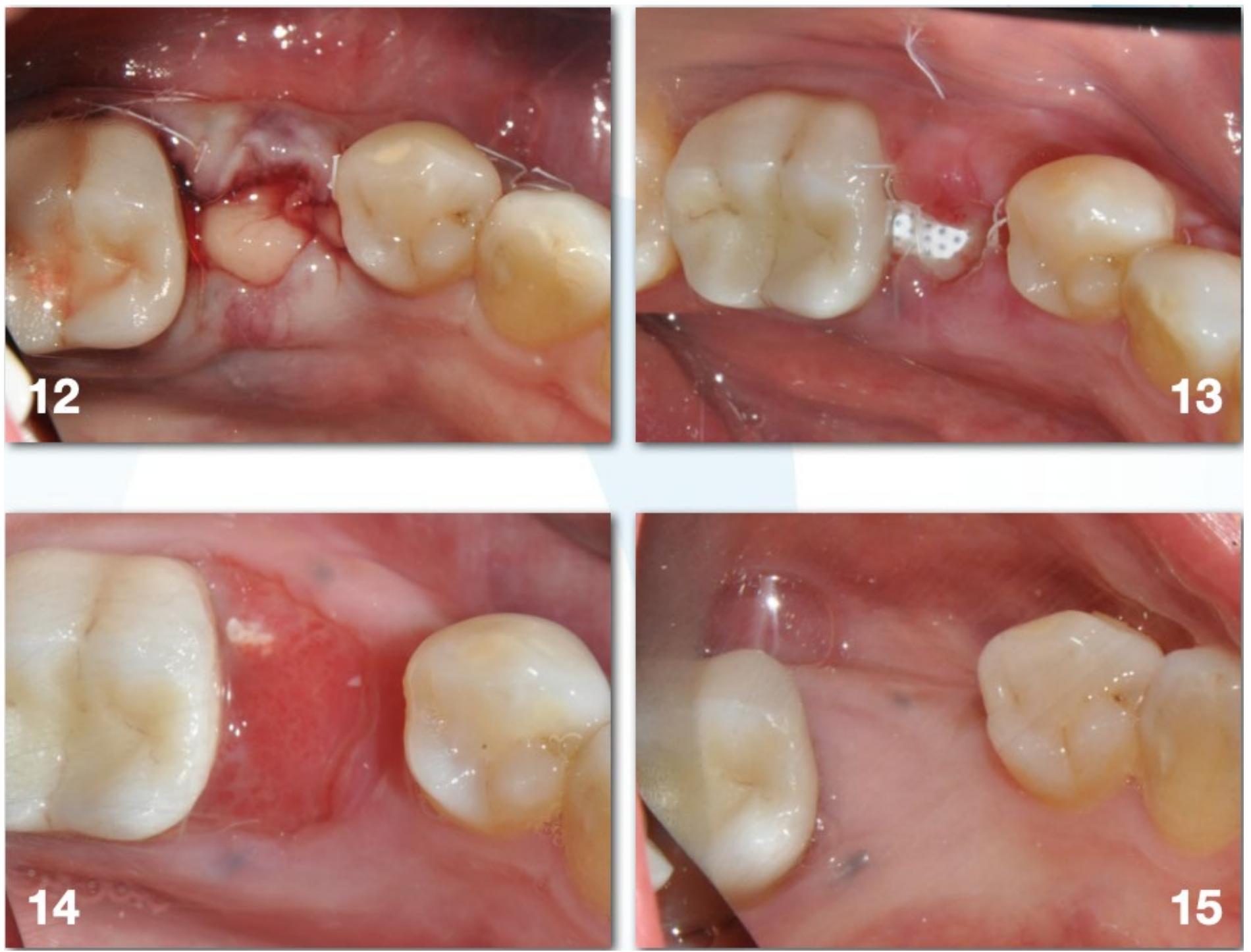

Figures 12-15. Healing evolution: after the first surgery / 2 weeks / 6 weeks (after removing the membrane) / 4 months

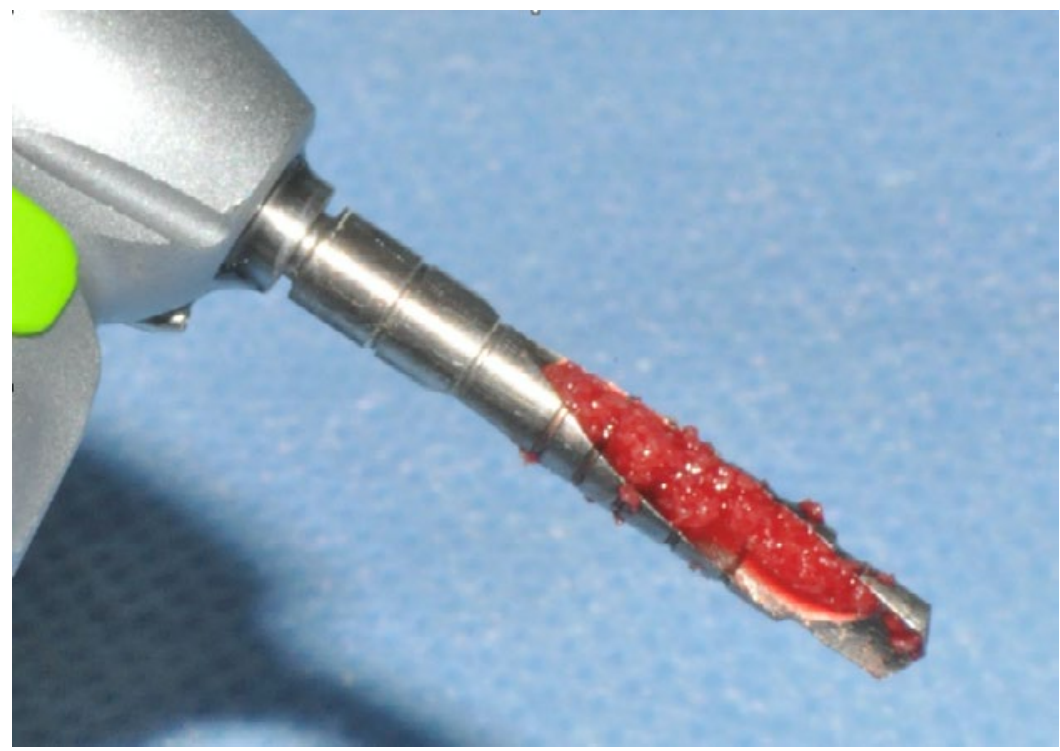

Figure 16. Quality of the regenerated bone (low revs drilling without irrigation) 


\section{CBCT comparison}

Figures 17-18-19 show a comparison between preoperative and postoperative (6 months after surgery) CBCTs: images are obtained by the superimposition tool (Anatomage In Vivo software), matching anatomical reference common points (in this case, remaining teeth, precision was $0.23 \mathrm{~mm}$ ).

The maximum horizontal gain is $13 \mathrm{~mm}$ and maximum vertical gain is $12 \mathrm{~mm}$

In second surgery the implant sites copiously bled: bone presented D3 density, the high Cone Beam CT Hunsfield Units (HU) measured in regenerated bone aren't indicative, because heavily influenced by not resorbed graft; however, insertion torques of implants were $45 \mathrm{~N}$ and $52 \mathrm{~N}$.

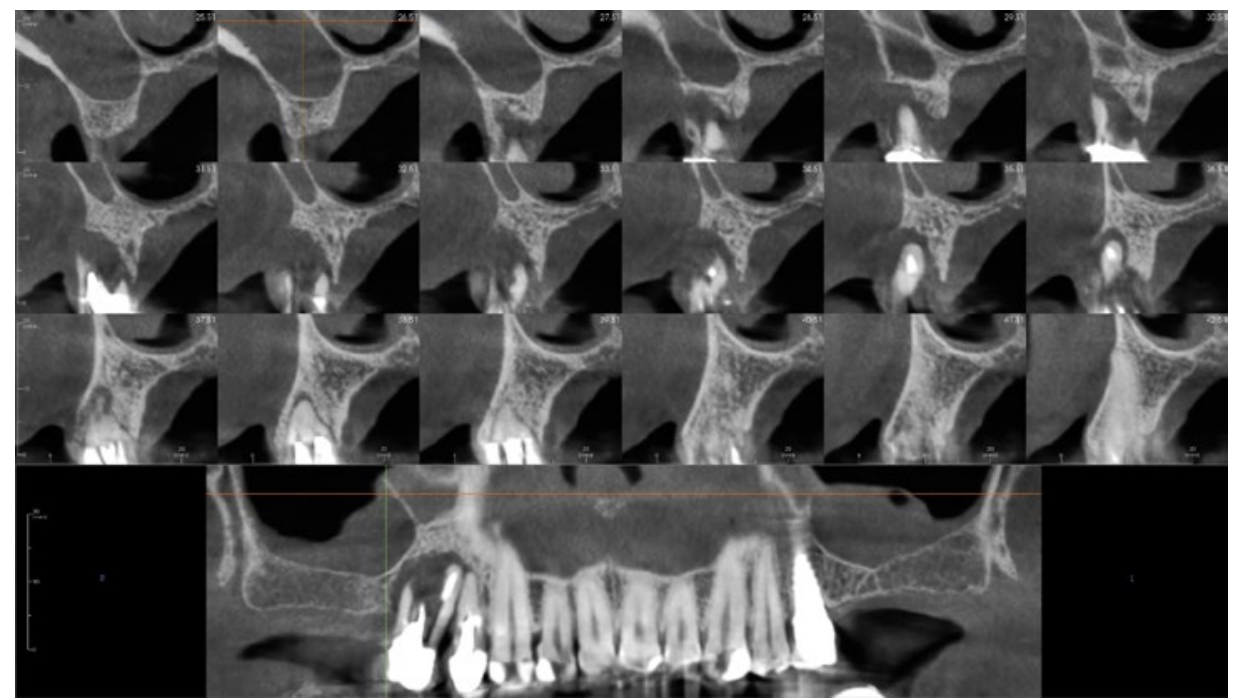

Figure 17. Initial CBCT

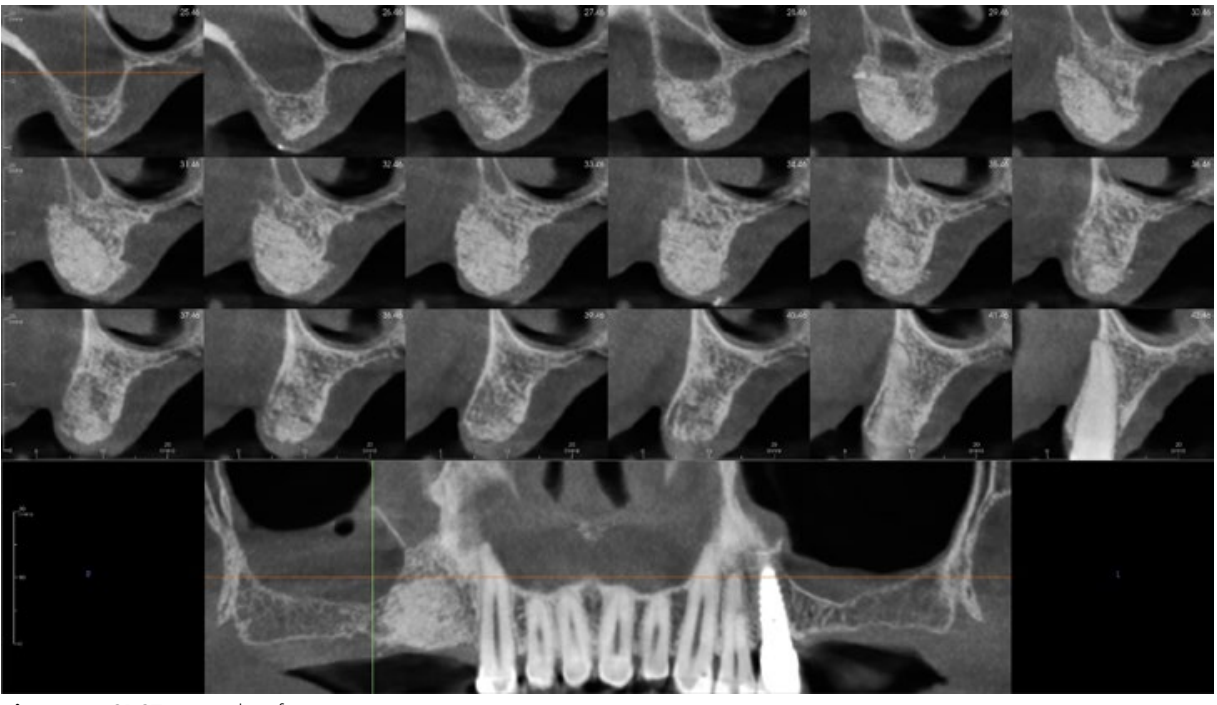

Figure 18. CBCT 6 months after surgery

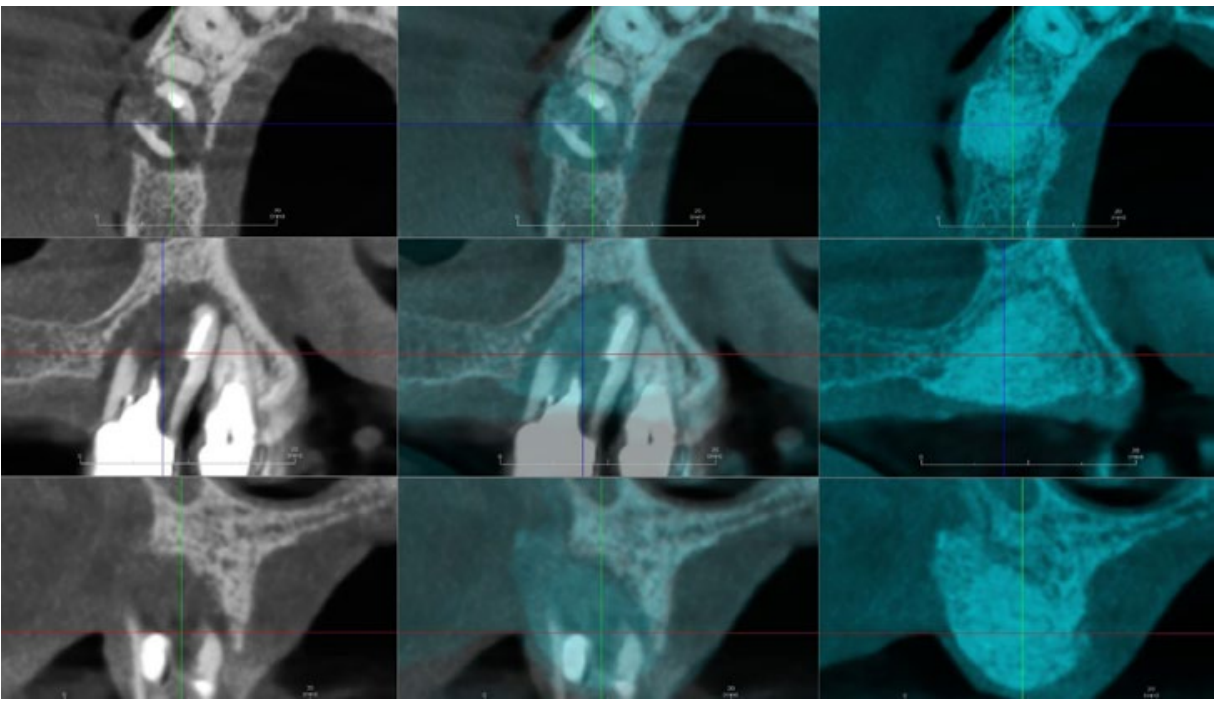

Figure 19. Comparison between CBCTs (initial and 6 months after surgery) 


\section{RESULTS AND CONCLUSION}

In 152 post-extractive cases we gain 100\% success rate, considering success the possibility to place implant at least $10 \mathrm{~mm}$ length and $3.7 \mathrm{~mm}$ diameter, but on average we placed implant $11,5 \mathrm{~mm}$ length and 4.3 diameter.

We registered some postoperative complications, but the final bone volume was always enough to place implant: in 12 cases the membrane was shaped too little for the site and tissue retraction exposed early its border, so we had to remove it before 6 weeks (in 3 cases after 10, 12 and 18 days and graft loss was remarkable); in 6 cases we have early infection of the graft, because interproximal border of membrane was exposed and/or the sutures are too relaxed to maintain strictly in position the PTFE membrane: in these cases we washed with hydrogen peroxide every 3-4 days and removed the membrane after 4 weeks (graft loss was important); in 25 cases we put an additional graft during implant placement, when the final bone volume obtained didn't allow to have almost $2 \mathrm{~mm}$ of vestibular regenerated bone, only to be sure preventing some tardive bone resorption and overcompensating some graft loss by complications.

The implant survival rate for 194 implants placed was 100\%, even if longer follow up is necessary to confirm these data, so we consider them as preliminary results.

Advantages for clinicians of our simplified protocol are an efficient and convenient alveolar preservation, with simple possibility to obtain horizontal and vertical bone augmentation; the amount and thickness of keratinized tissue simplifies the second surgery and allows to plan implant(s) prosthetically guided.

Our protocol does not use vertical incisions of the flap and horizontal incisions of periosteum, reducing postoperative symptomatology; the large amount of keratinized tissue avoids secondary surgical palatal site for connective graft.

In the eyes of patients, surgeries are less invasive, with more postoperative comfort; swelling is rare (3 cases in our study) and pain is totally kept under control by a short-term and low-dose analgesic therapy; patient compliance and satisfaction were very high.

Partial disadvantage is the little resorption of most superficial portion of the graft (1-3mm): it can be solved overcompensating by $2-3 \mathrm{~mm}$, especially in bigger volumes.

Complications can be prevented avoiding operative errors, especially incorrect or insufficient membrane shaping; in any event, they are manageable and remarkable graft loss is rare and simple to be recovered from the second surgery, thanks to the healthy and thick keratinized tissues obtained by secondary healing.

\section{CONFLICT OF INTEREST}

The author declares that there is no conflict of interest regarding the publication of this article.

\section{ACKNOWLEDGEMENTS}

Special thanks to Dr. Ilaria Barbalinardo DDS for precious help in searching and selecting the literature.

\section{REFERENCES}

1. Barber HD, Lignelli J, Smith BM, Bartee BK. Using a dense PTFE membrane without primary closure to achieve bone and tissue regeneration. J Oral Maxillofac Surg. 2007 Apr;65(4):748-52.

2. Zafiropoulos GG, Kačarević ZP, Oasim SSB, Trajkovski B. Open-Healing Socket Preservation with a Novel Dense Polytetrafluoroethylene (dPTFE) Membrane: A Retrospective Clinical Study. Medicina (Kaunas). 2020 Apr 28;56(5):216.

3. Barboza EP, Stutz B, Ferreira VF, Carvalho W. Guided bone regeneration using nonexpanded polytetrafluoroethylene membranes in preparation for dental implant placements--a report of 420 cases. Implant Dent. 2010 Feb;19(1):2-7.

4. Hoffmann O, Bartee BK, Beaumont C, Kasaj A, Deli G, Zafiropoulos GG. Alveolar bone preservation in extraction sockets using non-resorbable dPTFE membranes: a retrospective non-randomized study. J Periodontol. 2008 Aug;79(8):1355-69.

5. Lamb JW 3rd, Greenwell H, Drisko C, Henderson RD, Scheetz JP, Rebitski G. A comparison of porous and non-porous teflon membranes plus demineralized freezedried bone allograft in the treatment of class II buccal/lingual furcation defects: a clinical reentry study. J Periodontol. 2001 Nov;72(11):1580-7.

6. Simion M, Trisi P, Maglione M, Piattelli A. A preliminary report on a method for studying the permeability of expanded polytetrafluoroethylene membrane to bacteria in vitro: a scanning electron microscopic and histological study. J Periodontol. 1994 Aug;65(8):755-61.

7. Schmid J, Hämmerle CH, Olah AJ, Lang NP. Membrane permeability is unnecessary for guided generation of new bone. An experimental study in the rabbit. Clin Oral Implants Res. 1994 Sep;5(3):125-30.

8. Ronda M, Rebaudi A, Torelli L, Stacchi C. Expanded vs. dense polytetrafluoroethylene membranes in vertical ridge augmentation around dental implants: a prospective randomized controlled clinical trial. Clin Oral Implants Res. 2014 Jul;25(7):859-66.

9. Yamashita M, Horita S, Takei N, Sasada Y, Shibato W, Ishikawa Y, Takao K, Maki K, Funakoshi E. Minimally invasive alveolar ridge preservation/ augmentation procedure (open barrier membrane technique). Presented at the 2010 Research Forum Poster Session. Annual Meeting of the American Academy of Periodontology (AAP) in Honolulu, HI, October 30 - November 2, 2010.

10. Masuki H, Okudera T, Watanebe T, Suzuki M, Nishiyama K, Okudera H, Nakata K, Uematsu K, Su CY, Kawase T. Growth factor and pro-inflammatory cytokine contents in platelet-rich plasma (PRP), plasma rich in growth factors (PRGF), advanced platelet-rich fibrin (A-PRF), and concentrated growth factors (CGF). Int J Implant Dent. 2016 Dec;2(1):19.

11. Pitzurra L, Jansen IDC, de Vries TJ, Hoogenkamp MA, Loos BG. Effects of L-PRF and A-PRF+ on periodontal fibroblasts in in vitro wound healing experiments. J Periodontal Res. 2020 Apr;55(2):287-295.

12. Solakoglu Ö, Heydecke G, Amiri N, Anitua E. The use of plasma rich in growth factors (PRGF) in guided tissue regeneration and guided bone regeneration. A review of histological, immunohistochemical, histomorphometrical, radiological and clinical results in humans. Ann Anat. 2020 Sep;231:151528.

13. Brucoli M, Sonzini R, Bosetti M, Boffano P, Benech A. Plasma rich in growth factors (PRGF) for the promotion of bone cell proliferation and tissue regeneration. Oral Maxillofac Surg. 2018 Sep;22(3):309-313. 